3. Maffei, P. Contr. Obs. Asiago no. 136, 1963; no. 140, 1963.

4. Rosino, L. In press.

5. Götz, W. Veröff. Sternw. Sonneberg, 4, 245, 1958.

6. Herbig, G. Adv. Astr. and Astrophys., 1, 47, 1962.

7. Walker, M. C. R. Acad. Sci. Paris, 253, 383,$1961 ; R$. Obs. Bull. no. 82, 1964.

8. Herbig, G. Adv. Astr. and Astrophys., I, $87,1962$.

9. Haro, G. Astr. F., 65, 490, I960; Symposium on Stellar Evolution, La Plata, p. 37, I962.

ro. Herbig, G. Astrophys. F., I35, 736, 1962.

II. Joy, A. H. in Stellar Atmospheres, p. 66r, I96r.

\title{
4. FLARE STARS IN THE ORION NEBULA
}

\section{G. Haro}

A large number of plates have been obtained over the past ro years at the Tonantzintla Observatory in a search for flare stars; they are centred on the Trapezium and cover an area of 20 sq. degrees. Rosino and his colleagues have also searched for flare stars in the same field. Altogether, I2I stars with flare activity have now been discovered in this area. Their distribution on the sky is not random, but follows the general distribution of the $T$ Tauri-like stars in the region.

Our colour-magnitude diagram of $V$ vs. $B-V$ of the flare stars in Orion shows the following interesting feature: the flare stars appear abruptly at about $V \cong 13.5$, with a colour of about $+\mathrm{I} \cdot 0$ magnitude. Practically all the brightest flare stars lie above the main sequence; the horizontal departure from the main sequence is from several tenths of a magnitude to the blue of the main sequence up to nearly 4 magnitudes towards the red. To still fainter magnitudes, beyond $V=16.0$ about half of the flare stars lie on or above the main sequence, while the other half lie below.

The earliest spectral type of a flare star in the Orion Nebula is between Ko and K2. In NGC 2264, we found that the earliest was about $\mathrm{K}_{\mathrm{I}}$. Of course, there are many other flare stars in both aggregates as late as $\mathrm{M}_{2}$, or even later. As we pass from these young aggregates to older groups, we notice immediately that the spectral types of the brightest flare stars (at minimum light) found in these older organizations become later. For instance, in the Pleiades the brightest known flare stars are $\mathrm{K}_{5}$, while in Coma, Praesepe, the Hyades, and the solar neighbourhood all are of type M. On this evidence, it seems probable that the earliest type possible for a flare star is about $\mathrm{KI}$, which should mark in the $\mathrm{H}-\mathrm{R}$ diagram a left-hand boundary for the appearance of this kind of object. Perhaps there is also a right-hand boundary defined by stars of very low mass $(\leqslant 0.07 \odot)$ which, due to internal degeneracy, cannot remain on the main sequence.

In the Orion Nebula, there is a remarkable spread in the spectral types of the flare stars as well as in their vertical scatter both above and below the main sequence. This might be caused by a difference in the ages and in the evolutionary states of the flare stars in this aggregate. The location of such stars well below the main sequence-if real-constitutes an apparent discrepancy between observation and the conventional theory of gravitational contraction.

A good part of our observational results gives strong empirical support to Hayashi-type contraction, and conversely, Hayashi's theory seems to explain and clarify some of our observations. For example, if one draws in a theoretical H-R diagram that curve which is the locus of the intersection of the purely convective tracks with the purely radiative ones for a range of 
stellar masses, Poveda (I) found that the vertical line tangent to this curve lies exactly at type $\mathrm{K}_{\mathrm{I}}$, which is, as I have said, the earliest spectral type found by us for the flare stars in Orion. Another example: the $\mathrm{H}$-burning ages of the various aggregates and the ages derived for the flare stars in them by the Hayashi theory, are of the same order. And finally, our empirical evidence which relates the UV Ceti stars of the solar neighbourhood to the flare stars in young aggregates can easily be understood through the theoretical work of Hayashi and his collaborators and of Kumar, if we accept the general picture of gravitational contraction for star formation.

[Explanatory note by $G$. H. Herbig: It may be useful to clarify here a matter of nomenclature that has given rise to some confusion among non-specialists in this field: namely, the difference between flare and flash variables. The flare stars are well-known objects: found originally among the dMe stars (although a few show no strong emission spectrum) in the solar neighbourhood, there is no question but that these flare stars have no connection whatsoever with nebulosity at the present time. They are often known as UV Ceti variables, after one of the most active examples. About I953, however, Haro and his collaborators began to find in young aggregates such as the Orion Nebula and the Taurus dark clouds a type of low-luminosity variable that, photometrically, showed the same kind of short-lived outbursts as the flare stars. But these spectra showed clearly that although the stars were of $K$ and $M$ type, almost without exception they had no strong $\mathrm{H}$ or Ca II emission between outbursts, unlike most UV Ceti variables. Since these non-emission objects were clearly associated with the nebulae, it was felt best to keep them clearly separate, at least in name, from the flare variables of the solar neighbourhood until some clearer evidence of their physical connection with UV Ceti-like objects could be found. Hence these rapid variables in the nebulae were given the name flash stars. It is now beginning to appear, mainly as the result of work by Haro and his colleagues, that in older aggregates which are now completely free of nebulosity, one still finds variables of the flash type, giving rise to the old suspicion that the dMe flare stars of the solar neighbourhood are no more than low-mass examples of the flash family that long ago escaped from their parent aggregates. Consequently, if this concept survives, the necessity for a distinction between flash and flare stars may soon disappear.]

\section{REFEREN CE}

I. Poveda, A. Nature, 202, I319, I964.

\section{PHYSICAL CONDITIONS IN THE GAS OF THE NEBULA}

\section{T. K. Menon}

Since the early days of astronomical spectroscopy, a large number of spectroscopic and photometric investigations have been made of the Orion Nebula. Among the more detailed ones may be mentioned those due to Campbell and Moore (I), Wyse (2), Wurm and Rosino (3), Osterbrock and Flather (4), Wilson, Münch, Flather, and Coffeen (5), Aller and Liller (6), Menon (7), and Boyce (8). These investigations have provided us with a wealth of data using spectroscopic, spectrophotometric, photoelectric, photographic and radio frequency techniques. In this paper we shall summarize the information on the physical conditions in the gas of the Orion Nebula obtained from the above studies. 